\title{
MODELLING THE SATELLITE COMMUNICATION LINKS WITH ORTHOGONAL FREQUENCY-DIVISION MULTIPLEXING
}

\author{
Volodymir Kharchenko ${ }^{1}$, Bo Wang ${ }^{2}$, Andrii Grekhov ${ }^{3}$, Anna Leschenko ${ }^{4}$ \\ ${ }^{1,3,4}$ Dept Air Navigation Systems, Air Navigation Institute, National Aviation University, Kyiv, Ukraine \\ ${ }^{2}$ College of Economics and Management, Ningbo University of Technology, Ningbo, China
}

Submitted 18 January 2014; resubmitted 29 August 2014;

first published online 28 January 2015

\begin{abstract}
Global technologies of data transmission in real time are developing constantly. Therefore, modelling of messages transmission through satellite constellations is an actual problem. In this paper the original model of a communication channel 'Vehicle-to-Satellite-to-Ground Station' based on principles of the IEEE 802.11a standard is designed using MatLab Simulink software. The model allows simulating data transmission with data rate from 6 to $54 \mathrm{Mbit} / \mathrm{s}$ using adaptive modulation. Two types of channels were analysed for uplink/downlink modulation - a free space path losses with phase/frequency offset and Additive White Gaussian Noise (AWGN) link. On the base of this model, channel integrity was investigated and dependences of a Signal-Noise Ratio (SNR) on free space path losses, antennas diameters, number of OFDM symbols and satellite transponder noise temperature were received.
\end{abstract}

Keywords: satellite communication channel; vehicle; satellite transponder; ground station; convolutional coding; free space loss; phase/frequency offset; Viterbi decoder; transponder noise temperature; antenna diameter.

\section{Introduction}

Remote satellite communications allow obtaining and sending info and data messages over long distances very simple and easy (Elbert 2008). Satellites successfully serve traditional - telephony and broadcasting - markets, covering a large geographic area with transmission in a single beam. Currently, there is a demand for twoway broadband access in the major geographic areas, which are not served by telecommunication infrastructure. Therefore, satellite broadband technology is critical to the spread of multimedia services and applications throughout the world (Gómez 2002). This technology already exists and allows creating a huge number of interconnected broadband networks in space available for a dynamic and competitive market (Roddy 2006).

A new generation of applications has appeared requiring high bandwidth, and satellites satisfy these needs in a high throughput. Satellite systems are optimized for services such as internet access, virtual private networks and personal access. It is expected that the number of satellite broadband subscribers in world will grow to $\sim 6$ million in 2020 (ITU 2012).
The only servicing the Asia-Pacific satellite with high bandwidth is THAICOM-4 (IPSTAR). This is the satellite with multiple spot beams and direct relay board without regenerative payload. It provides a full range of nationwide broadband satellite services in Australia, Cambodia, China, India, Indonesia, Japan, Malaysia, Myanmar, New Zealand, Philippines, Republic of Korea, Thailand and Vietnam and has 18 gateways located in the countries it serves. Custom beams operate in the $\mathrm{Ku}$-band, and gateway services - Ka-band. IPSTAR has 84 focused beam, a special form of the three beams and the maximum bandwidth $45 \mathrm{Gbit} / \mathrm{s}$. Satellite provides a variety of services for state, commercial and industry sectors: controlled cell transit lines for virtual private networks, satellite news gathering, telephone service in rural areas, retail broadband, communications for the reconstruction of disaster and emergency communications and distance learning (IPSTAR 2013).

For wide dissemination of satellite communications it is necessary to eliminate two disadvantages. The first drawback - the delay (the time required to send and receive messages). It depends on the distance between

Current original article has been corrected since first publishing online. Please see the statement of correction (doi:10.3846/16484142.2016.1161942 of the corrigendum).

Corresponding author: Andrii Grekhov

E-mail: grekhovam@ukr.net

Copyright @ 2015 Vilnius Gediminas Technical University (VGTU) Press

http://www.tandfonline.com/TRAN 
the satellite and the Earth. The second is caused by the satellite signals, which can be loosened under the influence of rain or atmospheric conditions. To address these shortcomings, there are technical ways related to satellite orbits and to the available radio spectrum - two elements occupying a central place in the satellite system (Osborne, Xie 1999).

In a traditional satellite communication technology a single beam is used to cover all continents and regions. Recently, several narrowly focused beams are used allowing the reuse of frequencies to increase a bandwidth of 20 times or more compared to conventional satellites. Despite the higher costs associated with the technology of the focused beam, a channel estimated value is much lower than the technology based on a special shape of the beam (Manual for ICAO... 2007; HighSpeedSat 2008).

Remote satellites are used as a service for automating the processes of planning. Manufacturers and distributors of various goods, companies in wholesale and retail trade, including the online shopping, scope of service (vending machines, couriers, garbage removal and maintenance), national logistics and postal companies will benefit from the use of remote satellites (Marlink 2013; Intelsat 2012). Using satellite communication channels it is possible to build instantly the best routes for each car, distribute the load on cars taking into account their suitability, save the time and exclude errors (human factor), integrate with any Client Relationship Management (CRM) or accounting system for the transfer of orders, predict the exact arrival of a car to the point of the destination, to access the system from any computer or tablet (Malone 1999).

Mobile satellite telecommunication technologies evolve in the direction of the creation and development IP networks, new technologies using spectrally efficient signal-code constructions based on the use of M-PSK frequency modulations, M-QAM modulations, technologies of Orthogonal Frequency Division Multiplexing (OFDM) and Multiple-Input Multiple-Output (MIMO), which combines the spatial diversity and OFDM (Dahlman et al. 2013; Hanzo et al. 2010).

Wireless access is the main destructive factor for the digital channel in a broadband and it is an interference from multipath reception. This type of interference is very characteristic for radio reception in cities with buildings of different heights due to multiple signal reflections from buildings and other structures. A radical solution to this problem is the use of OFDM technology, which is specifically designed to deal with interference of multipath reception. In OFDM technology serial data stream is converted to a large number of parallel streams (sub-carriers), each of which is transferred on a separate carrier (Chang 1966).

Frequency spacing between adjacent carriers of OFDM spectrum is selected from the condition of the possibility to release individual carriers in the demodulator. OFDM is characterized by strong overlap of adjacent sub-carriers spectra, which reduces the frequency spacing and in the same time increase the density of transmitting digital information. Due to the orthogonal demodulation method of subcarriers the compensation of interference from adjacent frequencies takes place, despite the fact that their sidebands are overlapping (Ohno, Giannakis 2002).

Due to the fact that a large number of parallel streams are used, the symbol duration in parallel flows is substantially longer than in the serial data stream. This allows delaying an estimation of received symbols in the decoder for the time during which change the radio channel parameters due to the echoes will be stopped, and the channel will be stable.

OFDM sub-carrier symbol timeslot is divided into two parts - a guard interval, during which the evaluation of the character in the decoder is not performed and the working symbol interval, during which a decision about the received symbol value is made (Ma et al. 2003).

Modelling of satellite telecommunications channels without error-control coding was realized previously in our papers (Kharchenko et al. 2012a, 2012b, 2013a, 2013b).

The aim of this paper is to investigate conditions for effective messages transmission:

- to design the model of communication channel 'Vehicle-to-Satellite-to-Ground Station' with error-control coding using MatLab Simulink software;

- on the base of this model investigate a channel integrity and receive dependences of a signalnoise ratio (SNR) on a free space path loss, antennas diameters, number of OFDM symbols and satellite transponder noise temperature;

- to analyse two types of uplink and downlink channels (with a free space path loss and phase/ frequency offset and with Additive White Gaussian Noise (AWGN).

\section{1. 'Vehicle-to-Satellite-to-Ground Station' Link Design}

The model is based on principles of the IEEE 802.11a standard (ANSI/IEEE Std 802.11; Clark 2003), which is commonly used today for providing wireless connectivity in the home, office and commercial establishments. The communication range depends on the transmitter power, the receiver design, jamming and signal path. It is necessary to take into account the interaction of radio waves with ordinary objects of the building, such as the walls, metal structures, and even people in order to provide the necessary range of signal propagation. Scope of satellite systems with powerful transmitter and perfect antenna can reach the necessary range for communication with the low-orbit satellites.

Created model supports data rates $6,9,12,18,24$, 36,48 , and $54 \mathrm{Mb} / \mathrm{s}$, uses adaptive modulation and coding over a satellite communication channel with free space path losses, whereby the simulation varies the data rate dynamically.

The model (Fig. 1) for adaptive modulation in free space satellite communication channel 'Vehicle-to-Satellite-to-Ground Station' consists of 'Vehicle Transmitter' 


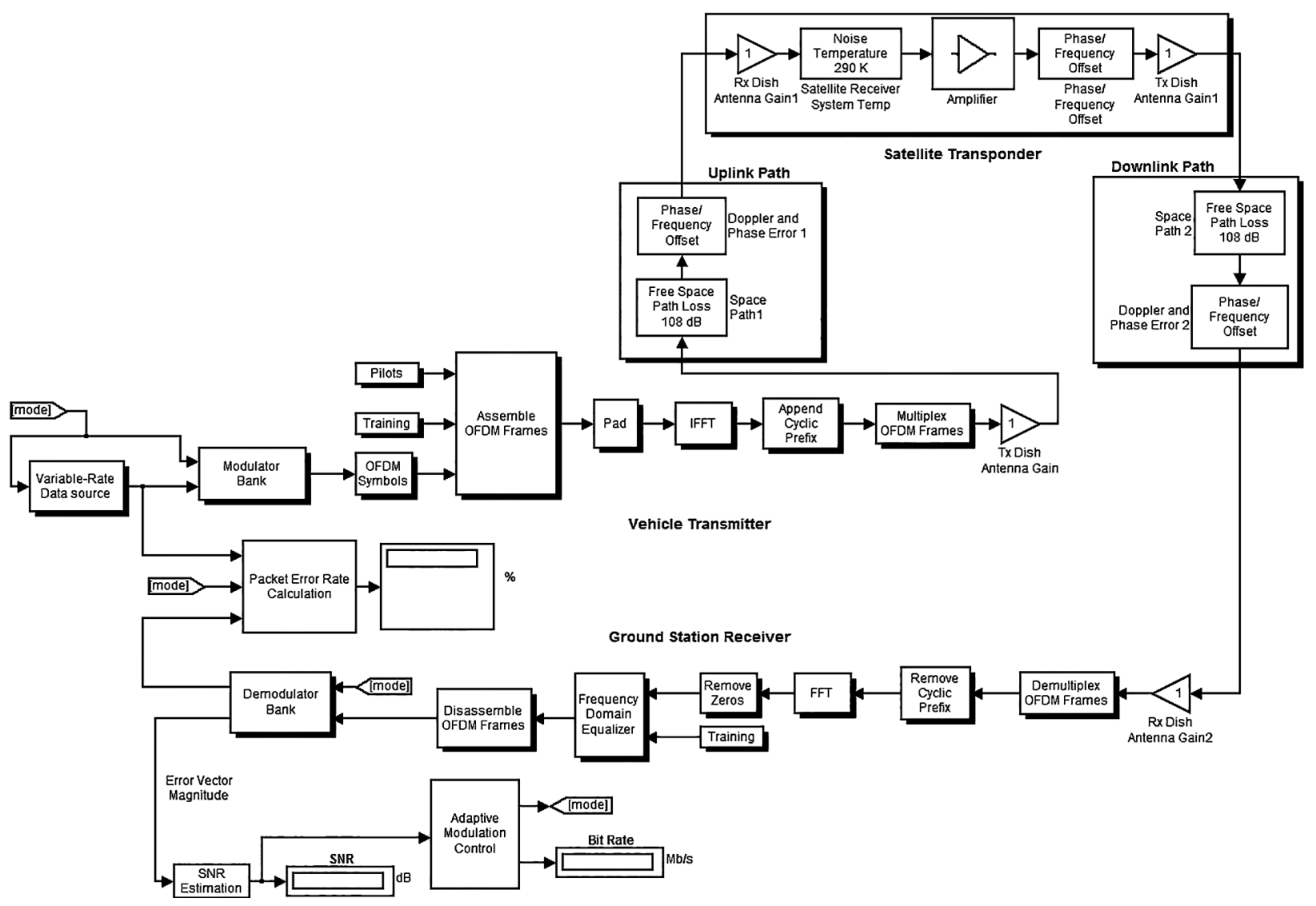

Fig. 1. Model of 'Vehicle-to-Satellite-to-Ground Station' link

(Variable-Rate Data Source, Modulator Bank, OFDM Transmitter, Transmitter Dish Antenna Gain), 'Uplink Path' (Free Space Path Loss, Phase/Frequency Offset), 'Satellite Transponder' (Receiver Dish Antenna Gain, Satellite Receiver System Temperature, Complex Baseband Amplifier, Phase/Frequency Offset, Transmitter Dish Antenna Gain), 'Downlink Path' (Free Space Path Loss, Phase/Frequency Offset), 'Ground Station Receiver' (Receiver Dish Antenna Gain, OFDM Receiver, Demodulator Bank), 'Packet Error Rate Calculation block', 'SNR Estimation' and 'Adaptive Modulation Control'.

Parameter settings for the model are the following: Viterbi traceback depth is 34 (Viterbi 1971), hysteresis factor for adaptive modulation $(\mathrm{dB})$ is 3 , numbers of OFDM symbols per transmit block are 20 and 1000 , number of OFDM symbols in training sequence is 4, Low-SNR thresholds ( $\mathrm{dB})$ vector [ [ $\left.\begin{array}{lllllll}10 & 11 & 14 & 18 & 22 & 26 & 28\end{array}\right]$ where 10 is for BPSK $1 / 2,11$ - for BPSK 3/4, 14 - for QPSK 1/2, 18 - for QPSK 3/4, 22 - for 16-QAM 1/2, 26 - for 16-QAM 3/4 and 28 - for 64-QAM 2/3.

Low-SNR thresholds parameter is a seven-element vector that indicates how the simulation should choose a data rate based on the SNR estimation. The model has eight modes, each associated with a particular modulation scheme and convolutional code. The seven thresholds are the boundaries between eight adjacent regions that correspond to the eight modes. Ideally, the simulation should use the highest-throughput mode that achieves a desired (zero) packet error rate. Determining appropriate thresholds often involves running the simulation multiple times, varying the values of the Low-SNR thresholds parameter.

The communication system in this model performs such tasks as:

- generation of random data at a bit rate that varies during the simulation;

- coding, interleaving, and modulation using one of several schemes specified in the standard;

- OFDM transmission using 52 subcarriers, 4 pilots, 64-point FFTs (Fast Fourier Transform), and a 16-sample cyclic prefix;

- physical layer convergence protocol preamble modelled as four long training sequences.

In the 'Vehicle Transmitter' Variable-Rate Data Source block generates random data and includes binary source. Binary data are created according to the SNR estimation at the receiver.

Modulator Bank block performs convolutional coding and puncturing using code rates of $1 / 2,2 / 3$ and 3/4; data interleaving; BPSK, QPSK, 16-QAM and 64-QAM modulations.

Padding block changes the dimension of input matrix along its columns, rows, or both of them according to the specified values. Each row is equal to one subcarrier. Thus, rows are in the frequency domain and columns are in the time domain.

OFDM transmitter comprises Pilots, Training, OFDM symbols, Assemble OFDM Frames, Pad, IFFT 
(Inverse Fast Fourier Transform), Cyclic Prefix, and Multiplex OFDM Frames block.

In order to create the OFDM symbol a serial to parallel block is used to convert $N$ serial data symbols into $N$ parallel data symbols. Then, each parallel data symbol is modulated with different orthogonal frequency subcarriers, and added to an OFDM Symbol. All modulated subcarriers are added together to create the OFDM symbol. This is done by an Inverse Fast Fourier Transformation (IFFT). The advantage of using IFFT is that the system does not need $N$ oscillators to transmit $N$ subcarriers. Assemble OFDM Frames is applied in order to insert the pilot and training symbols into the OFDM symbols. Four pilots are inserted between the assemble subcarriers and then training sequence is added to the subcarriers.

In the 'Uplink (Downlink) Path' Free Space Path Loss block simulates the loss of signal power due to the distance between the aircraft uplink transmitter and the satellite transponder receiver. The block reduces the amplitude of the input signal by an amount that is determined by the Loss $(\mathrm{dB})$ Parameter.

Phase/Frequency Offset block applies a frequency and phase offset to the input signal.

In the 'Satellite Transponder' the Satellite Receiver System Temperature block simulates the effects of thermal noise on a complex, baseband signal. Modelling was provided for two values of effective satellite and ground station receiver systems noise temperatures $20 \mathrm{~K}$ (very low noise level) and $290 \mathrm{~K}$ (typical noise level). Satellite repeater or amplifier is a complex baseband model of amplifier with noise. Only linear amplifier was considered during investigation with gain equalling $1 \mathrm{~dB}$.

Satellite Receiver System Temperature adds receiver thermal noise to complex baseband signal.

In the 'Ground Station Downlink Receiver' the Viterbi Decoder block decodes input symbols to produce binary output symbols. Unquantized decision type parameter was used. The receiver performs the reverse operations of the transmitter, with additional training tasks.

Packet Error Rate Calculation block shows the packet error rate as a percentage and should always equal 0 during investigations.

SNR Estimation block estimates the SNR based on the error vector magnitude.

Adaptive Modulation Control takes into accounts Low-SNR thresholds, Hysteresis factor, and Bit rates. Adaptive modulation systems improve the rate of transmission. The implementation of adaptive modulation is according to the channel information that is present at the transmitter. The method of making adaptive modulation in this model is according to the estimated SNR, a bit rate will be specified and then data source generates binary data according to the specified data rate in adaptive modulation control.

The error rate calculation block calculates the bit error rate, by comparing the received data with transmitted data.

The Transmitter (Receiver) Dish Antenna Gain block multiplies the input by a constant value (gain).
Dependencies of a SNR and modulation mode on transmitting and receiving antennas diameter at various free path losses were obtained using vectors $\left[d_{1}, d_{2}\right]$ for each pair 'transmitter-receiver'. The first element in the vector $\left[d_{1}, d_{2}\right]$ represents the transmitting antenna diameter (in meters) and is used to calculate the gain in the Transmitter Dish Antenna Gain block. The second element represents the receiving antenna diameter and is used to calculate the gain in the Receiver Dish Antenna Gain block. Diameters of all antennas (transmitting antenna on a vehicle, receiving and transmitting antennas on a satellite, receiving antenna on a ground station) were changed simultaneously.

\section{Satellite Channels Performance Simulation}

For the communication channels of the first type with a free space path loss and phase/frequency offset dependencies of a SNR on free path losses for different modulation modes, antenna diameters, transponder amplifier linear gain and transponder noise temperature are shown in Fig. 2. For this modelling the following parameters in the model were set up:

- phase/frequency offsets in uplink/downlink and satellite transponder are equal to zero;

- the gain of linear amplifier in satellite transponder was taken $1,3,12$;

- free space path losses were taken in such a way that packet error rate always equals zero;

- number of OFDM symbols in training sequence is 4 .

The number of OFDM symbols per transmit block was taken 20 and 1000 .

As follows from Fig. 2 for the communication channels of the first type changing of all antennas diameter and different transponder noise temperatures has significant influence on character of dependencies. At the same time the number of OFDM symbols per transmit block practically does not change the dependencies.

For comparison of a free space path loss channel with a AWGN channel, the same model was taken, but uplink/downlink was substituted by AWGN block to simulate an AWGN channel. The AWGN Channel block adds white Gaussian noise to the input signal.

As follows from Fig. 3 for the communication channels of the second type changing of all antennas diameter has significant influence on character of dependencies. At the same time the number of OFDM symbols per transmit block practically does not change the dependencies.

In the presence of an arbitrary phase/frequency offsets introduced by the uplink/downlink and satellite transponder the demodulator sometimes is unable to tell which constellation point is which. End signal constellations for two types of communication channels are shown in Figs 4 and 5 for the specific case when conditions for signals propagation are almost similar. Nevertheless, the type of a communication channel plays a major role in determining the nature of the signal constellation. 


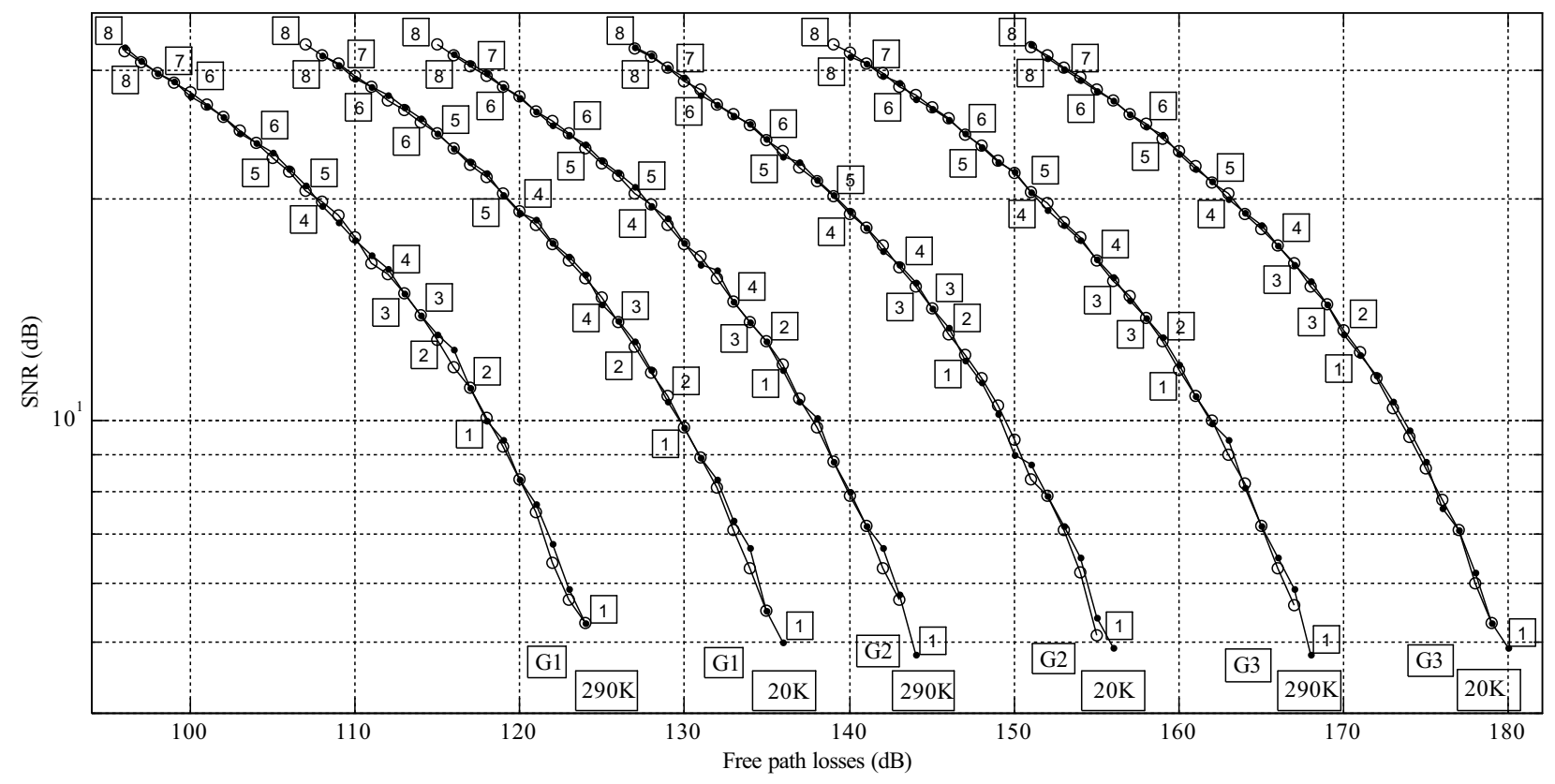

Fig. 2. Dependence of SNR on free path losses for different modulation modes ( 1 - BPSK $1 / 2,2$ - BPSK $3 / 4,3$ - QPSK $1 / 2$, 4 - QPSK 3/4, 5 - 16-QAM 1/2, 6 - 16-QAM 3/4, 7 - 64-QAM 2/3, 8 - 64-QAM 3/4), antenna diameters and transponder noise temperatures: dots - number of OFDM symbols per transmit block is 20, circles - number of OFDM symbols per transmit block is 1000; antennas gains: $G_{1}=1\left(d_{1}=d_{2}=d_{3}=d_{4}=0.03 \mathrm{~m}\right), G_{2}=3\left(d_{1}=d_{2}=d_{3}=d_{4}=0.1 \mathrm{~m}\right)$,

$$
G_{3}=12\left(d_{1}=d_{2}=d_{3}=d_{4}=0.4 \mathrm{~m}\right)
$$

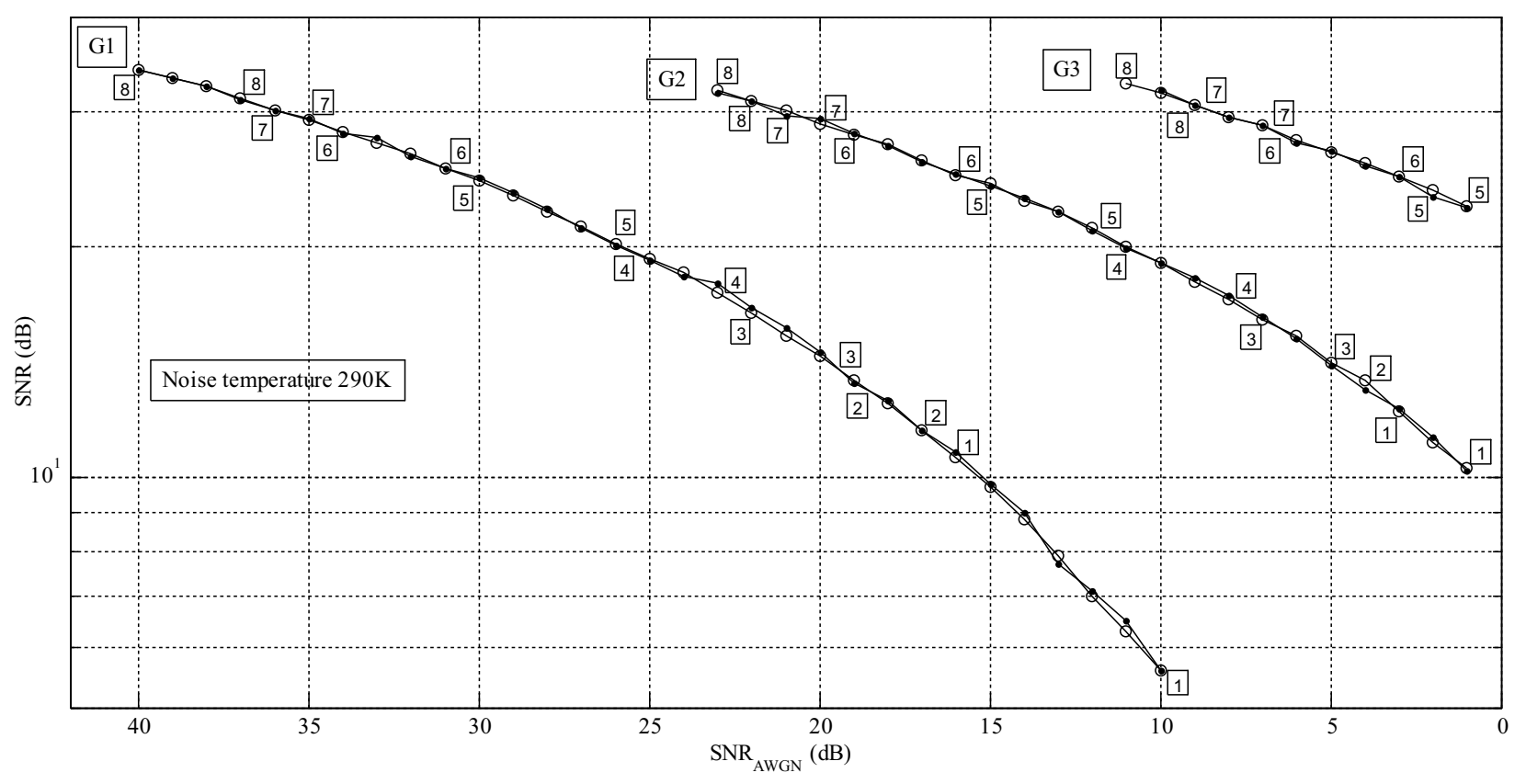

Fig. 3. Dependence of SNR on $\mathrm{SNR}_{\mathrm{AWGN}}$ for different modulation modes (1 - BPSK 1/2, 2 - BPSK 3/4, 3 - QPSK 1/2, 4 - QPSK 3/4, 5 - 16-QAM 1/2, 6 - 16-QAM 3/4, 7 - 64-QAM 2/3, 8 - 64-QAM 3/4), antenna diameters and transponder noise temperature $290 \mathrm{~K}$ : dots - number of OFDM symbols per transmit block is 20 , circles - number of OFDM symbols per transmit block is 1000; antennas gains: $G_{1}=1\left(d_{1}=d_{2}=d_{3}=d_{4}=0.03 \mathrm{~m}\right), G_{2}=3\left(d_{1}=d_{2}=d_{3}=d_{4}=0.1 \mathrm{~m}\right)$,

$$
G_{3}=12\left(d_{1}=d_{2}=d_{3}=d_{4}=0.4 \mathrm{~m}\right)
$$




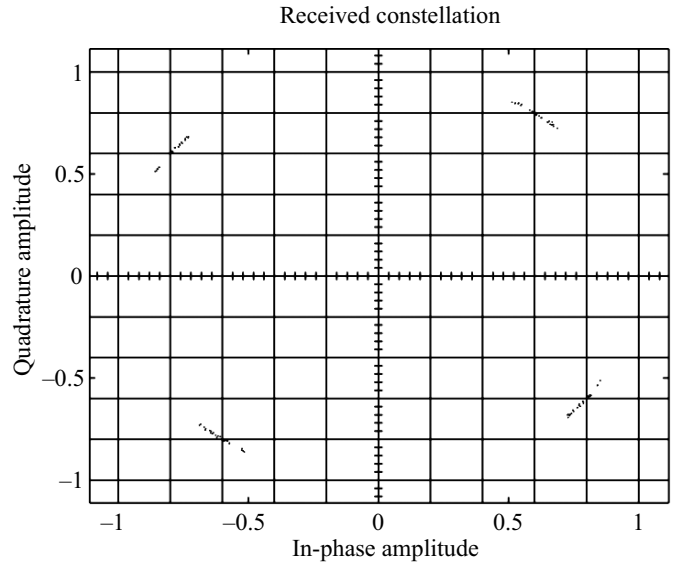

Fig. 4. End signal constellation for the communication channels of the first type with a free space path loss and phase/ frequency offset: free path losses are $50 \mathrm{~dB}$ in the uplink/downlink; phase offsets are $40^{\circ}$, frequencies shifts are $150 \mathrm{~Hz}$ in the uplink/downlink and satellite transponder; number of OFDM symbols per transmit block is 20 ; antennas gains are $G=1$; $\mathrm{SNR}=16.5 \mathrm{~dB}$; bit rate $12 \mathrm{Mb} / \mathrm{s}$; satellite transponder noise temperature $290 \mathrm{~K}$

\section{Conclusions}

During the modelling process for transmitting messages via low-orbit satellite constellation the original model of a communication channel 'Vehicle-to-Satellite-toGround Station' was built using MatLab Simulink software.

Two types of uplink and downlink channels with a free space path loss and phase/frequency offset and AWGN were investigated.

Dependencies of a SNR on free space path losses, antennas diameter, number of OFDM symbols and satellite transponder noise temperature were received and analysed.

Signal changes were analysed by means of active windows-indicators which allowed defining constellations of the transmitted and received signals (Figs 4 and 5).

This model can be used as the basic model for investigation of communication between many vehicles and ground stations using several satellites.

This paper covers evaluation of 'Satellite-to-Aircraft' link performance ('Vehicle-to-Satellite-to-Ground Station'), which can be used for self-separation of unmanned aircraft systems (in particular for remotely piloted aircraft).

\section{References}

ANSI/IEEE Std 802.11, 1999 Edition (R2003). Information Technology - Telecommunications and Information Exchange between Systems - Local and Metropolitan Area Networks Specific Requirements. Part 11: Wireless LAN Medium Access Control (MAC) and Physical Layer (PHY) Specifications. 513 p. http://dx.doi.org/10.1109/IEEESTD.2003.95617

Chang, R. W. 1966. Synthesis of band-limited orthogonal signals for multichannel data transmission, Bell System Technical Journal 45(10): 1775-1796.

http://dx.doi.org/10.1002/j.1538-7305.1966.tb02435.x

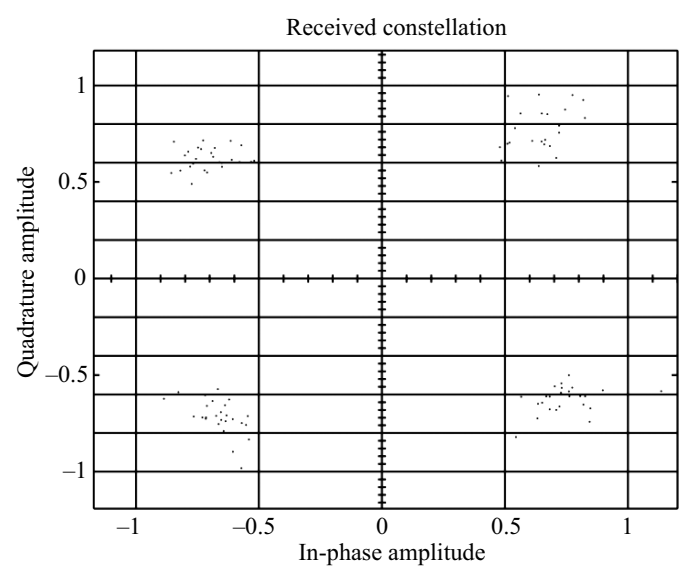

Fig. 5. End signal constellation for the communication channels of the second type with AWGN: $\mathrm{SNR}_{\mathrm{AWGN}}=18 \mathrm{~dB}$ in the uplink/downlink; phase offset is $40^{\circ}$, frequency shift is $150 \mathrm{~Hz}$ in the satellite transponder; number of OFDM symbols per transmit block is 20; antennas gains are $G=1$; $\mathrm{SNR}=16.7 \mathrm{~dB}$; bit rate $18 \mathrm{Mb} / \mathrm{s}$; satellite transponder noise temperature $290 \mathrm{~K}$

Clark, M. 2003. IEEE 802.11a WLAN Model. Available from Internet: http://www.mathworks.com/matlabcentral/ fileexchange/3540-ieee-802-11a-wlan-model

Dahlman, E.; Parkvall, S.; Skold, J. 2011. 4G: LTE/LTE-Advanced for Mobile Broadband. 2nd edition. Academic Press. $544 \mathrm{p}$.

Elbert, B. R. 2008. Introduction to Satellite Communication. 3rd edition. Artech House. 447 p.

Gómez, J. M. 2002. Satellite Broadcast Systems Engineering. Artech House. 224 p.

Marlink. 2013. Astrium Services Opens Rotterdam Based Global Logistics Centre. Press Releases. Available from Internet: http://www.marlink.com/news-astrium-services-opensrotterdam-based-global-logistics-centre.html

Hanzo, L. L.; Akhtman, Y.; Wang, L.; Jiang, M. 2010. MIMOOFDM for LTE, WiFi and WiMAX: Coherent Versus NonCoherent and Cooperative Turbo Transceivers. Wiley-IEEE Press. 692 p.

HighSpeedSat. 2008. Iridium Satellites. Available from Internet: http://www.highspeedsat.com/iridium-satellite.php

Intelsat. 2012. Logistics. Intelsat General Corporation (IGC). Available from Internet: http://www.intelsatgeneral.com/ category/tags/logistics

IPSTAR. 2013. IPSTAR Fact Sheet. Available from Internet: http://www.ipstar.com/pdf/IPSTARfactsheet.pdf

ITU. 2012. ITU Broadband Reports. International Telecommunication Union (ITU). Available from Internet: http://www. itu.int/ITU-D/treg/publications/bbreports.html

Kharchenko, V.; Barabanov, Y.; Grekhov, A. M. 2013a. Modeling of ADS-B data transmission via satellite, Aviation 17(3): 119-127.

http://dx.doi.org/10.3846/16487788.2013.840057

Kharchenko, V.; Barabanov, Y.; Grekhov, A. 2013b. Modelling of 'satellite-to-aircraft' link for self-separation, Transport 28(4): 361-367.

http://dx.doi.org/10.3846/16484142.2013.864699

Kharchenko, V. P.; Barabanov, Y. M.; Grekhov, A. M. 2012a. Modelyuvannya suputnykovogo aviacijnogo zv'yazku, Visnyk Nacional'nogo Aviacijnogo Universytetu - Proceedings of National Aviation University (1): 5-13. (in Ukrainian). 
Kharchenko, V. P.; Barabanov, Y. M.; Grekhov, A. M. 2012b. Modelyuvannya suputnykovogo kanalu peredachi ADS-B povidomlen', Visnyk Nacional'nogo Aviacijnogo Universytetu - Proceedings of National Aviation University (3): 9-14. (in Ukrainian).

Ma, X.; Giannakis, G. B.; Ohno, S. 2003. Optimal training for block transmissions over doubly selective wireless fading channels, IEEE Transactions on Signal Processing 51(5): 1351-1366. http://dx.doi.org/10.1109/TSP.2003.810304

Malone, R. 1999. QUALCOMM: Managing Information in Motion. Available from Internet: http://www.inboundlogistics. $\mathrm{com} / \mathrm{cms} /$ article/qualcomm-managing-information-inmotion

Manual for ICAO Aeronautical Mobile Satellite (Route) Service. Part 2-Iridium. Draft v4.0. 2007. 77 p. Available from Internet: http://www.icao.int/safety/acp/Inactive\%20working\%20groups\%20library/ACP-WG-M-Iridium-8/IRDSWG08-IP05\%20-\%20AMS(R)S\%20Manual\%20Part\%20 II\%20v4.0.pdf

Ohno, S.; Giannakis, G. B. 2002. Optimal training and redundant precoding for block transmissions with application to wireless OFDM, IEEE Transactions on Communications 50(12): 2113-2123.

http://dx.doi.org/10.1109/TCOMM.2002.806547

Osborne, W. P.; Xie, Y. 1999. Propagation characterization of LEO/MEO satellite systems at 900-2100 MHz, in 1999 IEEE Emerging Technologies Symposium. Wireless Communications and Systems, 12-13 April 1999, Richardson, TX, USA, 21.1-21.8. http://dx.doi.org/10.1109/ETWCS.1999.897339

Roddy, D. 2006. Satellite Communications. 4th edition. McGraw-Hill Professional. 636 p.

Viterbi, A. J. 1971. Convolutional codes and their performance in communication systems, IEEE Transactions on Communication Technology 19(5): 751-772.

http://dx.doi.org/10.1109/TCOM.1971.1090700 